\title{
On the Physical Mechanism Underlying PC1 and Baldwin Effect in Quasars
}

\author{
Xiao-Bo Dong ${ }^{1}$, Ting-Gui Wang ${ }^{1}$, Jian-Guo Wang ${ }^{2,1}$, Xiaohui Fan ${ }^{3}$, \\ Huiyuan Wang ${ }^{1}$, Hongyan Zhou ${ }^{1}$, and Weimin Yuan ${ }^{2}$ \\ ${ }^{1}$ Center for Astrophysics, University of Science and Technology of China \\ Email: xbdong@ustc.edu.cn \\ ${ }^{2}$ Yunnan Observatory, Chinese Academy of Sciences, Kunming, Yunnan 650011, China \\ ${ }^{3}$ Steward Observatory, The University of Arizona, Tucson, AZ 85721, USA
}

Keywords. galaxies: active, quasars: emission lines, quasars: general

Recent work (Baskin \& Laor 2004; Dong et al. 2009a,b) suggests that the Eddington ratio $\left(\ell \equiv L / L_{\mathrm{Edd}}\right)$ is the origin of all the significant first-order object-to-object variations of quasar spectral properties from the zeroth-order similarity of AGN spectra; specifically, this includes the PC1 of Boroson \& Green (1992), the classic or inverse Baldwin effect (Baldwin 1977), and even blueshifting (i.e., blue asymmetry) of high-ionization emission lines (Dong et al. 2009c).

To understand such unification, we shift our docus from the detailed physics of the accretion process (microphysics) to "statistical physics" (macrophysics) of the broad-line clouds (Dong et al., in preparation; cf. Korista 1999). With more realistic constraints (e.g., the distribution of the number of line-emitting clouds with $\ell$ and other physical parameters), we believe that the first-order regularities and even the second-order effects of quasar emission lines may be reproduced exactly by future "locally optimally-emitting cloud" models (LOC; Baldwin et al. 1995).

On one hand, for clouds gravitationally bound around the central engine, there is a lower limit on the column density $N_{\mathrm{H}}$ that is set by and roughly scales with $L / L_{\mathrm{Edd}}$ (Dong et al. 2009a). On the other hand, in accretion-powered radiation systems such as quasars, $L / L_{\mathrm{Edd}}$ is closely related to $\dot{m}$; thus the increase in $L / L_{\mathrm{Edd}}$ requires an increase in the gas supply. It is reasonable to conclude that at least a significant part of the gas required originates in the line-emitting clouds. Thus, as $L / L_{\mathrm{Edd}}$ increases, the total mass of line-emitting clumpy gas increases. Combining the above two factors, the distribution of the line-emitting clouds is ultimately regulated by $L / L_{\mathrm{Edd}}$.

Note that the strength of high-ionization lines (e.g., C IV) and optically thick lines (e.g., Ly $\alpha$ and $\mathrm{Mg}$ II) is roughly proportional to the summed area of the illuminated surface of line-emitting clouds while that of low-ionization, optically thin lines (e.g., narrow-line and broad-line optical Fe II) is roughly proportional to the summed volume of the clouds. Thus, generally, the EW of high-ionization lines and optically thick lines correlates negatively with $L / L_{\mathrm{Edd}}$ whereas that of low-ionization, optically thin lines correlates positively. The exact $\log E \mathrm{~W}-\log L / L_{\mathrm{Edd}}$ slope for a particular emission line depends on both the cloud distribution and the atomic physics - the latter is the very physics underlying the LOC model.

\section{References}

Baldwin, J. A. 1977, ApJ, 214, 679

Baldwin, J. A., et al. 1995, ApJ, 455, 119

Baskin, A. \& Laor, A. 2004, MNRAS, 350, L31

Boroson, T. A \& Green, R. F. 1992, ApJS, 80, 109

Dong, X.-B., Wang, T., Wang, J., Fan, X., Wang, H., Zhou, H., \& Yuan, W. 2009a, ApJ, 703, L1

Dong, X., et al. 2009b, submitted to ApJ [arXiv: 0903.5020]

Dong, X., et al. 2009c, in The Origin and Physical Mechanism of the Ensemble Baldwin Effect, ed. W. Wang, Z. Yang, \& Z. Chen (San Francisco: ASP), p. 83

Korista, K. T. 1999 in Quasars and Cosmology, (San Francisco: ASP), p. 165 